\title{
La cambiante economía de la Industria del turismo
}

\section{John Urry}

\section{Introducción}

La relación entre la mirada turistica y las industrias que se han desarrollado para atenderla es extremadamente problemática.

Inicialmente debe señalarse que casi todos los servicios brindados a los turistas tienen que entregarse en el mismo momento y lugar en que se les produce (véase Urry 1987). En consecuencia, la calidad de la interacción social entre el proveedor del servicio -como el mozo, la azafata y el recepcionista del hotel- y los consumidores forma parte del "producto" comprado por los turistas. Si algunos aspectos de dicha interacción social son insatisfactorios (un mozo descortés, una azafata que no sonrie o un recepcionista malcriado), entonces lo que se compra es, en efecto, un producto de servicio diferente. El problema se debe al hecho de que la producción de dichos servicios para el consumidor no puede llevarse a cabo integramente tras bastidores, fuera de la mirada de los turistas. Éstos no pueden evitar ver algunos aspectos de la industria que intenta servirles. Pero además, los turistas tienden a tener una expectativa alta de lo que debieran recibir, dado que "irse de viaje" es un evento dotado de un significado especial. Las personas esperan lo extraordinario y por lo tanto serán excepcionalmente criticas de los servicios que parezcan socavar dicha calidad.
Otros rasgos de las industrias turisticas probablemente crearán dificultades para los productores de dichos servicios. Éstos no pueden suministrarse en cualquier lugar: tienen que producirse y consumirse en lugares muy particulares. De hecho, parte de lo que se consume es el lugar en donde se encuentra situado el productor del servicio. Si el lugar particular no transmite los significados culturales apropiados, la calidad del servicio especifico puede muy bien quedar menoscabada. Hay, por lo tanto, una "fijeza espacial" crucial en lo que respecta a los servicios turísticos. En años recientes se ha dado una competencia enormemente acentuada para atraer turistas. En lo que respecta a Gran Bretaña, hubo una "europeización" del mercado turístico y una "globalización" cada vez mayor. De modo tal que mientras que los productores son en gran medida fijos espacialmente -en tanto que tienen que suministrar servicios particulares en lugares específicos-, los consumidores son cada vez más móviles, capaces de consumir servicios turísticos sobre una base global. La industria es inevitablemente competitiva puesto que casi cualquier lugar del mundo podría muy bien actuar como objeto de la mirada turística. Dichos servicios son inherentemente intensivos en mano de obra, por lo cual los empleadores buscarán minimizar los costos laborales. Para conseguir eso se emplean diversas estrategias, pero por lo menos algunas de ellas dañarán en parte o socavarán totalmente la naturaleza extraordinaria de la mirada turistica de los consumidores. 
El énfasis en la calidad de la interacción social entre los productores y los consumidores de los servicios turísticos significa que los desarrollos en la industria no son explicables simplemente en términos de determinantes "económicos". Como se mostrará posteriormente, es asimismo necesario examinar una serie de cambios culturales que transforman las expectativas de las personas sobre qué desean ver, qué importancia se debe atribuir a dicha mirada y qué efectos tendrá esto en los proveedores de los servicios turísticos relevantes. Esta es una industria que siempre ha requerido niveles considerables de participación e inversión pública, y en años recientes ello se ha incrementado a medida que todo tipo de lugares intentan construir o reforzar su posición como objetos favorecidos por la mirada del turista. La economia del turismo no puede entenderse separada del análisis de los desarrollos culturales y políticos a encontrarse más adelante en este libro. del mismo modo que el trabajo en las industrias turisticas no puede comprenderse desligado de las expectativas culturales que rodean la compleja entrega de dichos servicios. Las relaciones laborales en estas industrias están significativamente definidas culturalmente.

En este capitulo se dirigirá la atención sobre algunos de los desarrollos recientes más evidentes en lo que en forma no estricta podemos llamar la cambiarte economía politica de la industria del turismo. L a siguiente sección hace un breve recuento del concepto de bienes posicionales, el concepto económico más importante usado para explicar la economía del turismo, antes de pasar a la cambiante industria turistica del Reino Unido señalando en particular sus tendencias a la globalización - y a los cambios más importantes en la economia política del turismo extranjero.

\section{Los límites sociales del turismo}

El economista Mishan (1969; véase Urry 1990, asi como el Journal of Sustainable Tourism y la revista de propaganda Tourism in Focus) presenta una de las versiones más claras de la tesis de que hay límites fundamentales a la escala del turismo contemporáneo. Estos limites se derivan de los inmensos costos de congestión y apiñamiento. En la década de 1960 Mishan (1969: 140) escribió perceptivamente del "conflicto de intereses ... entre, de un lado, los turistas, las agencias de turismo, las industrias del tráfico y los servicios anexos, para no mencionar los gobiernos ansiosos por incrementar sus reservas de divisas extranjeras, y del otro a todos aquellos que se preocupan por conservar la belleza natural".

Mishan citaba el ejemplo del lago Tahoe, cuya vida vegetal y animal ha sido destruida por las aguas servidas generadas por los hoteles construidos a lo largo de sus orillas. Un ejemplo posterior seria la forma en que el coral alrededor de islas turisticas como Barbados viene muriendo, gracias al bombeo de aguas servidas no tratadas al mar desde los hoteles en las playas, y porque los nativos retiran tanto plantas como peces del coral para vendérselos a los turistas.

Mishan también señala que hay un conflicto de intereses entre las generaciones actuales y futuras, que se deriva de la forma en que se fijan los precios de los viajes y el turismo. El costo del turista marginal no tiene en cuenta los costos de congestión adicionales impuestos por el visitante extra. Estos últimos costos comprenden los efectos generalmente no deseados de playas atiborradas, falta de paz y quietud, la destrucción del paisaje y el uso de combustibles fósiles que contribuyen al calentamiento global. Es más, el turista ecológicamente sensible sabe que no se ganará nada retrasando una visita al lugar en cuestión; en todo caso lo opuesto sucederá. Hay un fuerte incentivo para ir lo antes posible: para disfrutar la vista no malograda antes de que las multitudes lleguen alli (como sucede actualmente con el destino emergente de La Habana). En la siguiente afirmación podemos ver que la perspectiva de Mishan (1969: 141) es la de una persona horronizada por las consecuencias del turismo de masas, en oposición al viaje individual: "en una carrera competitiva por descubrir a la multitud repleta de dinero todos los lugares que alguna vez tuvieron paz y quietud, y fueron de maravilla, hermosura e interés histónico, el negocio del turismo está, en efecto, destruyéndolos literal e irrevocablemente". Su elitismo de clase media y edad madura jamás está lejos de la superficie. Por ejemplo, él sostiene que son los "jóvenes y crédulos" los que creen las fantasias soñadas por la industria del turismo (uno se pregunta cuál seria su opinión sobre la lbiza contemporánea). 
El principal argumento de Mishan es que la propagación del turismo masivo no produce una democratización del viaje. Se trata de una ilusión que destruye los mismos lugares que están siendo visitados. Ello se debe a que el espacio geográfico es un recurso estrictamente limitado. Mishan (1969: 142) dice: "lo que unos cuantos pueden gozar en libertad, la multitud necesariamente destruye para si". A menos que se llegue a un acuerdo internacional (jél sugirió la prohibición inmensamente radical de todos los viajes aéreos internacionales!), la siguiente generación heredará un mundo casi privado de lugares de "belleza natural no perturbada" (Mishan 1969: 142). De modo que permitir al mercado desarrollarse sin regulación alguna tiene el efecto de destruir los mismos lugares que son objetos de la mirada turística. Un número creciente de estos lugares están sufriendo el mismo patrón de auto-destrucción. Un balneario que recientemente se ha dado en pensar que está dañado de este modo es St. Tropez, el lugar inicialmente vuelto famoso por Brigitte Bardot. Ella afirma que éste viene siendo barrido por "una marea negra de porquería humana"; que los turistas "son mediocres, sucios, de malos modales y malcriados"; y que piensa "dejárselo a los invasores" (véase Rocca 1989; sobre el crimen y el turismo véase Mawby, Brunt y Hambly 2000).

Este tipo de argumento pesimista es criticado por Beckerman (1974: 50-52), quien hace dos observaciones útiles. En primer lugar, la preocupación por los efectos del turismo de masas es esencialmente una ansiedad de "clase media" (al igual que muchas otras preocupaciones ecologistas). Ello se debe a que los realmente ricos "están bastante seguros de las masas en sus centros vacacionales sumamente caros, o en sus yates o islas privados, o en propiedades apartadas" (Beckerman 1974: 50-51). En segundo lugar, la mayoria de los grupos afectados por el turismo de masas en realidad sí se benefician con él, e incluso algunos de los visitantes pioneros, que entonces encuentran servicios disponibles que no se podian conseguir antes, cuando el número de visitantes era algo pequeño. De ahi que Beckerman (1974: 51) señale el "egoísmo estrecho de quejas como la de Mishan".
El desacuerdo en torno a los efectos del turismo de masas recibe más peso teórico con la tesis de Hirsch (1978; véase también la compilación de Ellis y Kumar 1983) sobre los limites sociales del crecimiento. Su punto de partida es similar al de Mishan: él señala que la liberación individual mediante el ejercicio de la elección del consumidor no hace que dichas elecciones sean liberadoras para todas las personas en conjunto (Hirsch 1978: 26). Le interesa, en particular, la economía posicional. Este término se refiere a todos los aspectos de los bienes, servicios, trabajo, posiciones y otras relaciones sociales que son o bien escasos, o sujetos a la congestión o apiñamiento. Por lo tanto, la competencia es una suma cero: a medida que una persona cualquiera consume más del bien en cuestión, otra se ve forzada a consumir menos. La oferta no puede incrementarse, a diferencia del caso de los bienes materiales en que los procesos de crecimiento económico pueden producir más con facilidad. El consumo que las personas hacen de los bienes posicionales es inherentemente relacional. La satisfacción derivada por cada persona no es infinitamente expandible, sino que depende de la posición del consumo de cada uno con respecto al de otros. Esto puede denominarse "competencia coercionada". Ellis y Heath (1983: 16-19) definen esto como una competencia en la cual el status quo no es una opción. Usualmente se asume en economía que los intercambios en el mercado son voluntarios y que las personas eligen libremente si entran o no en la relación de intercambio. Sin embargo, en el caso del consumo forzoso ellas en realidad no cuentan con semejante elección. Uno tiene que participar aun cuando al final del proceso de consumo no necesariamente esté mejor. Podemos resumir esto con la frase "uno tiene que correr con mayor rapidez para quedarse quieto". Hirsch cita el ejemplo de la suburbanización. La gente se muda a los suburbios para escapar de la congestión de la ciudad y estar más cerca de la tranquilidad del campo. Pero a medida que el crecimiento económico continúa los suburbios se congestionan más, se expanden y de este modo sus habitantes originales quedan tan lejos del campo como se hallaban originalmente. Buscarán, por lo tanto, nuevas viviendas suburbanas más cerca 
Artículos

del campo y asi sucesivamente. Las acciones individualmente racionales de otros hacen que uno esté peor y no es posible evitar participar en el proceso de salto de rana. Nadie queda mejor a lo largo del tiempo debido a dicho consumo coercionado.

Hirsch sostiene que buena parte del consumo tiene caracteristicas similares al caso del suburbanismo, a saber, que la satisfacción que las personas derivan de ello depende de las elecciones de consumo de otros. Esto puede verse con mayor claridad en el caso de ciertos bienes que son escasos en sentido absoluto. Los ejemplos aquí citados son los "viejos maestros" o "el paisaje natural", en donde el incremento en el consumo de uno lleva a la reducción del consumo de otra persona (pero véase Ellis y Heath 1983: 6-7). Hirsch asimismo considera los casos en los cuales hay una "escasez social directa": bienes de lujo o tal vez esnobs que se gozan porque son raros o costosos, y cuya posesión indica status social o buen gusto Los ejemplos aqui incluyen las joyas, una residencia en una parte especifica de Londres, o ropa de marca. Un tercer tipo que Hirsch considera es el de la "escasez social incidental": bienes cuyo consumo brinda una satisfacción que está influida por la amplitud de su uso. Los ejemplos negativos en este caso incluyen la compra de un automóvil sin ningún incremento en la satisfacción debido a la creciente congestión, ya que todos hacen lo mismo; y la obtención de calificaciones educativas sin ninguna mejoria en el acceso a las posiciones de liderazgo porque todos han estado adquiriendo credenciales similares (Ellis y Heath 1983: 10-11).

Es bastante fácil sugerir ejemplos del turismo que coinciden con estas diversas formas de escasez. En lo que respecta al primero, la costa mediterránea está en condición de escasez absoluta alli donde el consumo de uno es a expensas del de otro. En cuanto al segundo, evidentemente hay muchos destinos vacacionales que se consumen, no porque sean intrinsecamente superiores, sino porque transmiten gusto o status superior. Para los europeos, el Caribe o el Lejano Oriente serian ejemplos actuales, aunque esto cambiará a medida que se transformen los patrones mismos del turismo masivo. $Y$ tercero, hay muchas locaciones turis- ticas en donde la satisfacción de las personas depende del grado de congestión. Hirsch (1978: 167) cita a un profesional de clase media que observó que el desarrollo de los vuelos fletados baratos a un país antes "exótico" significaba que "ahora que puedo costear el venir aquí sé que será arruinado".

Aunque he presentado estos distintos tipos de bienes posicionales identificados por Hirsch, las distinciones entre ellos no son del todo sostenibles y se fusionan entre sí. Es más, hay una serie de grandes dificultades en su argumentación. En el caso del turismo, resulta ambiguo qué se quiere decir con consumo. ¿Es la capacidad para mirar un objeto particular, de ser necesario en compañia de muchos otros? ¿O es poder mirar sin que otros estén presentes? ¿Acaso es poder contratar alojamiento por un breve periodo con una vista del objeto que está a la mano? ¿O es la capacidad para poseer propiedades con una vista del objeto cercano? El problema surge debido a la importancia que la mirada tiene para la actividad turística. Después de todo ella es visual, puede literalmente tomar medio segundo y los otros servicios brindados son en cierto sentido periféricos al proceso fundamental de consumo, que es captar la vista. Esto significa que la escasez involucrada en el turismo es más compleja de lo que Hirsch acepta. Una estrategia seguida por la industria turística fue iniciar nuevos proyectos que permitieron que un número enormemente incrementado de personas contemplara el mismo objeto. Entre los ejemplos tenemos la construcción de enormes complejos hoteleros fuera de la costa; el desarrollo de vacaciones fuera de la temporada alta para que la misma vista pueda ser apreciada durante todo el año; el diseño de vacaciones para distintos segmentos del mercado, de modo tal que una gama más amplia de visitantes potenciales pueda ver el mismo objeto; y el desarrollo de alojamientos de tiempo compartido para que las instalaciones puedan utilizarse todo el año.

Es más, la noción de escasez resulta problemática por otras razones. Comenzaré señalando la distinción entre la capacidad de carga física de una locación turística y su capacidad percibida (véase Walter 1982). En el primer término está claro cuándo un sendero de mon- 
taña literalmente no puede tomar más caminantes puesto que ha sido erosionado y realmente ha desaparecido. Ello no obstante, incluso en este caso siguen habiendo miles de otros senderos de montaña que podrian recorrerse, de modo que la escasez solamente se aplica a este sendero que lleva a esta vista particular, no a todos los caminos en todas las montañas.

La noción de la capacidad percibida cambia la situación. A Walter (1982: 296) le interesa aqui la cualidad subjetiva de la experiencia turística. Aunque todavia se pueda recorrer el sendero fisicamente, éste ya no significa lo silvestre pristino que el espectador esperaba de la contemplación. De este modo se habria colmado su capacidad de carga perceptiva, pero no su capacidad fisica. Walter prosigue señalando que la capacidad perceptiva es inmensamente variable y depende de concepciones particulares de la naturaleza y de las circunstancias en que las personas esperan contemplarla. Nos cita el ejemplo de una montaña alpina. En tanto bien material, ella puede ser contemplada por su grandeza, belleza y adecuación con el pico alpino idealizado. Este bien casi no tiene límite. La montaña seguirá conservando estas cualidades, no importa cuantas personas la estén mirando. Sin embargo, la misma montaña puede ser vista como un bien posicional, como una suerte de santuario a la naturaleza que las personas desean gozar en soledad, sin que haya otras presentes. Un "consumo" solitario semejante muestra un buen gusto nada ambiguo (para estas distinciones véase Bordieu 1984). Hay, entonces, una forma "romántica" de la mirada turistica, en la cual el énfasis está en la soledad, la privacidad y la relación personal y semiespiritual con el objeto de la contemplación. Barthes (1972: 74) caracteriza este punto de vista tal como figura en la Guide Bleu; él habla de "esta promoción burguesa de las montañas, este viejo mito alpino ... sólo las montañas, barrancos, desfiladeros y torrentes ... parecen estimular la moral del esfuerzo y la soledad".

Walter (1982: 298) discute el ejemplo de Stourhead Park, en Wiltshire, que ilustra

La noción romántica de que el yo se encuentra, no en la sociedad sino en la contemplación solitaria de la natu- raleza. El jardín de Stourhead es el paisaje romántico perfecto, con angostos senderos serpenteando entre los árboles y rododendros, grutas, templos, un cottage gótico, todo esto alrededor de un lago de orillas con muchos recovecos ... El jardín está diseñado para que se camine a su alrededor maravillándose con la $\mathrm{Na}$ turaleza, y la presencia de otras personas inmediatamente comienza a perjudicar esto.

Al examinar a Mishan señalé su énfasis en que la "belleza natural no perturbada" constituye el objeto típico de la mirada turística. Sin embargo, ésta es en realidad sólo un tipo de mirada, la que denomino "romántica". Hay una alternativa: la mirada turística "colectiva", con características diferentes. He aquí la descripción que Walter (1982: 198) hace de Longleat, otra casa y jardin de Wiltshire:

Una gran casa señorial, colocada en un parque Capability Brown (1); los árboles fueron deliberadamente recortados ... para que pueda verse el parque desde la casa y ella desde aquel. En efecto, la casa es el punto focal del parque ... el folleto enumera veintiocho actividades y servicios ... Todas estas actividades y las multitudes resultantes encajan simpáticamente dentro de la tradición de la casa señorial; la vida del aristócrata era esencialmente pública antes que privada.

Estos lugares fueron diseñados como lugares públicos: se verían raros si estuviesen vacios. Son las otras personas las que los hacen. De este modo, la mirada colectiva requiere la presencia de un gran número de otras personas. Son ellas las que le dan al lugar una atmósfera o sensación de carnaval. Ellas indican que éste es el lugar donde estar y que uno no debiera estar en otro sitio. Y como vimos, uno de los problemas de los balnearios costeros británicos es que no hay suficientes personas para transmitir este mensaje. Como dice Walter (1982: 298), "Brighton o Lyme Regis en un día soleado de verano, con la playa enteramente para uno (1) Lancelot " Capability Brown (1715 - 1783) célebre jardinero paisajista ingles $\mathrm{N}$ del $\mathrm{T}$. 
mismo, serian una experiencia fantasmal", La presencia de otros turistas, personas como uno, es en realidad necesaria para el éxito de estos lugares, que dependen de la mirada turística colectiva. Este es también el caso de las grandes ciudades, cuya singularidad es su naturaleza cosmopolita. Es la presencia de personas de todo el mundo (turistas, en otras palabras) lo que da a las ciudades capitales su distintiva emoción y glamour (véase Walter 1982: 299).

Un gran número de otros turistas no generan únicamente congestión, como sugiere el argumento posicional. Su presencia brinda un mercado para los tipos de servicios que la mayoria de ellos están en realidad desesperados por adquirir, tales como alojamiento, comidas, bebidas, viajes y esparcimiento.

De este modo, los argumentos de Hirsch sobre la escasez y la competencia posicional se aplican fundamentalmente a aquellos tipos de turismo caracterizados por la mirada romántica. Donde se encuentra la mirada colectiva hay menos problemas con el apiñamiento y la congestión. $Y$ en efecto, el argumento de Hirsch descansa sobre la noción de que sólo hay un número limitado de objetos que pueden ser vistos por el turista. En años recientes se ha dado un enorme incremento en los objetos de la mirada turística, que caen mucho más allá de la "belleza natural no perturbada" de Mishan. Parte de la razón detrás de este incremento yace en el hecho de que los turistas contemporáneos son coleccionistas de contemplaciones que parecen no estar tan interesados en la visita repetida al mismo lugar aurático. La mirada inicial es lo que cuenta en lo que denomino la mirada espectadora.

Quienes realmente sí valoran la soledad y la mirada turistica romántica no ven esto simplemente como una forma de considerar la naturaleza. En lugar de ello intentan hacer que todos la sacralicen del mismo modo (véase Walter 1982: 300-303). El romanticismo, se ha difundido y generalizado, extendiéndose desde las clases medias altas, aunque la noción de la naturaleza romántica es un placer esencialmente inventado y variable. $Y$ cuanto más intentan sus adherentes difundir sus virtudes a otros, tanto más se minan las condiciones de la mirada romántica: "el turista romántico esta cavando su propia tumba si busca predicar a otros su propia religión" (Walter 1982: 301). La mirada romántica es un mecanismo importante que está ayudando a propagar el turismo a escala global, atrayendo a casi todos los países a su ámbito a medida que los románticos buscan objetos siempre nuevos de dicha mirada, y minimizando la diversidad a través de una ampliación de lo que Turner y Ash (1975) llaman la "periferia del placer".

La mirada turística contemporánea está cada vez más señalizada. Hay señales que identifican las cosas y lugares dignos de nuestra mirada. Esta señalización identifica un número relativamente pequeño de nudos turísticos. El resultado es que la mayoria de los turistas están concentrados dentro de un área sumamente limitada. Como dice Walter (1982: 302): "el nudo sagrado brinda un bien posicional que es destruido por la democratización". Él, por el contrario, favorece la idea de que hay "gemas a encontrarse en todo lugar y en todas las cosas ... no hay limite alguno a lo que puede encontrarse" (Walter 1982: 302). Debiéramos, nos dice, alejarnos de la tendencia a construir la mirada turística en unos cuantos lugares sagrados y selectos, y ser mucho más católicos en los objetos a los que podemos mirar. Esto indudablemente ha sucedido en años recientes, en especial con el desarrollo del turismo industrial, rural y del patrimonio cultural. Sin embargo, el análisis que Walter hace de la naturaleza de clase de la mirada romántica es persuasivo, y cierro esta sección sobre la teoría económica del turismo señalando su análisis cabalmente sociológico de lo penetrante de lo romántico, en oposición a la mirada colectiva, y el problema subsiguiente del bien posicional de muchas locaciones turisticas:

Los profesionales que se dedican a formar la opinión (autores de panfletos, profesores, el personal de las comisiones de distritos rurales, (2) son mayormente de clase media y es dentro de esta clase que el deseo romántico de bienes posicionales tiene su base principal. De este modo, la soledad romántica tiene auspiciadores in-

(2) Countryside Commission, organismo gubemamental británico encargado de proteger la campina inglesa. $\mathrm{N}$ del $\mathrm{T}$. 
fluyentes y recibe una buena publicidad. En cambio, el goce de la convivencia, la sociabilidad y de formar parte de una multitud, que es mayormente de la clase obrera, es frecuentemente mal visto por aquellos a quienes les preocupa la conservación del ambiente. Esto es infortunado, porque ello ... exalta una actividad al alcance únicamente de los privilegiados (Walter 1982: 303).

\section{La globalización y la economía del turismo}

Ya vimos que el balneario costero inglés inició su decadencia a mediados de la década de 1960, en el momento en que el turismo de masas se internacionalizaba, por lo menos en Europa. Ha seguido habiendo un crecimiento masivo de los flujos internacionales de turistas (véase WTO 2000a, 2000b). Esta internalización del turismo significa que no podemos explicar los patrones turisticos en una sociedad particular sin analizar los desarrollos que tienen lugar en la mayoria de los restantes paises. Su internacionalización, sobre todo en Europa, significa que toda locación turística puede ser comparada con aquellas situadas en el extranjero (en especial a través de la Internet). De modo que cuando las personas visitan algún lugar en su propio país están, en efecto, eligiendo no visitar uno en el extranjero. La internacionalización del turismo significa que todos los objetos potenciales de la mirada turística pueden ser colocados en una escala y comparados entre sí, ahora a menudo en forma más o menos instantánea a través de la Internet.

El resultado de esta internacionalización es que distintos países, o diferentes lugares dentro de un pais, pasan a especializarse en el suministro de tipos particulares de objetos a mirar. En las dos últimas décadas surgió una división internacional de las locaciones turísticas. Gran Bretaña ha pasado a especializarse en la historia y el patrimonio cultural, y esto afecta tanto lo que esperan los visitantes del extranjero, como aquello que atrae a los residentes del Reino Unido a pasar sus vacaciones dentro de él. Es más, esta internacionalización de las vacaciones se encuentra más desarrollada en es- te último lugar que en algunos otros países. Ello se debe en parte al desarrollo temprano e innovador del paquete o vacación inclusiva en Gran Bretaña, y en parte a la disponibilidad de muchas locaciones turísticas idóneas para atraer un gran número de turistas del extranjero. Así como la economía británica en general es una economía abierta, así también sucede con el turismo. Consideraré brevemente la naturaleza de la industria de los paquetes vacacionales antes de examinar las principales características de la industria de las vacaciones domésticas del Reino Unido.

Los operadores turísticos con base en él han vendido sus paquetes vacacionales o vacaciones inclusivas a un precio considerablemente más barato que en países comparables de Europa. En la década de 1980, eran los operadores turísticos con base en Gran Bretaña los que ofrecian los precios más bajos en la mayoria de los hoteles de España, Portugal y Grecia; dichas compañias fueron particularmente efectivas en reducir los costos unitarios y en generar un inmenso mercado para los viajes internacionales en el Reino Unido, y todavía existe un diferencial de precio significativo entre los precios de los paquetes vacacionales allí y en el resto de Europa (página web de Guardians Unlimited). Actualmente se venden al año alrededor de diecisiete millones de paquetes vacacionales (en comparación con unos ocho millones en 1983; BTA 2000). La razón principal por la cual las vacaciones inclusivas han tenido tal impacto en Gran Bretaña es el temprano surgimiento de compañías integradas -los operadores turísticos-, las cuales hicieron un uso espectacularmente exitoso de las nuevas tecnologias de transporte en jet y de los sistemas computarizados de reserva de pasajes (véase Reynolds 1989: 330-33).

En Gran Bretaña, un considerable nivel de concentración acompañó el desarrollo de la industria de los paquetes turísticos. De este modo hay cuatro grandes operadores: Airtours, First Choice, Thompson y Thomas Cook. Los dos últimos son ahora propiedad de compañias alemanas, Thompson de Preussag y Thomas Cook de $C$ \& N Touristic, en tanto que First Choice ha acordado una alianza estratégica con la compañia estadounidense Royal Caribbean 
(página web de Guardians Unlimited). Y sin embargo, aún hay muchas pequeñas compañias que brindan vacaciones inclusivas; 640 miembros de la ABTA tienen un movimiento de menos de $£ 6$ millones (English Tourism Council 2000). Sin embargo, la participación de las grandes compañias se ha incrementado constantemente hasta alcanzar alrededor del 57 por ciento (Chandler 2000: D5-9). Pareceria que esto se debe en parte a que han comenzado a atender tanto los destinos más exóticos -donde pueden bajar los precios mediante la compra al por mayor de camas de hotel- como los gustos más especializados de aquello que llamo el "post-turista". Thompson, por ejemplo, tuvo veinticuatro folletos orientados a distintos segmentos del mercado incluso en la década de 1980 (Williams 1988; The Economist, 27 de agosto de 1988).

Desde la formación de un único mercado europeo, los operadores turisticos de este continente trabajan en cada uno de los principales países a mayor escala. Eso ha incrementado la competencia y reducido el nivel de concentración dentro de un solo país, además de incrementar la toma y fusión de empresas más allá de las fronteras. $Y$ asimismo ha elevado el nivel de integración vertical, siendo los operadores también dueños de agencias de viaje, hoteles y líneas aéreas (véanse los detalles en Chandler 2000: D5-9). Sin embargo, en la última encuesta de clientes de vacaciones para Holiday Which? ¿Qué vacación?, se juzgó a estos grandes operadores como "los de peor rendimiento en la industria". Las seis mejores compañias fueron todas pequeñas compañias independientes. ¡De los grandes operadores, solamente Thompson logró evitar estar en la categoria de los diez peores! (página web de Guardians Unlimited).

Esta es una industria en la cual las nuevas tecnologias son particularmente apropiadas debido a los inmensos problemas de información y comunicaciones existentes. Los sistemas ahora permiten a los clientes "auto-servirse" pasajes aéreos y otros productos estandarizados. En efecto, hoy es posible visualizar una "agencia de viajes sin papel". Actualmente los consumidores pueden armar paquetes mucho más flexibles, una suerte de "rompecabezas" vacacional, o lo que la industria llama Free and Independent Tra- vel [Viaje Libre e Independiente] o FIT, en especial a través de Internet. Los desarrollos expertos en los sistemas permiten que el viajero potencial presente algunos parámetros del viaje deseado y luego permita a la computadora generar una serie de posibles productos de consumo.

Es más, parecería que con el incremento en el tiempo de esparcimiento la gente, los jóvenes en especial, está dejando de lado cada vez más el paquete vacacional algo estandarizado y buscando una variedad más amplia de formas de actividades recreativas, entre ellas los viajes independientes (Desforges 1998). La proporción de vacaciones inclusivas tomadas por los residentes del Reino Unido cayó del 38 por ciento de todas las visitas al extranjero en 1983, a 34 por ciento en 1998 (BTA 2000: 52 53). Ello está obligando a los operadores turísticos a desarrollar tipos más flexibles de arreglos de viaje. Ha habido un marcado incremento en la compra únicamente de ... seats-only flights, debido en parte a la demanda de más flexibilidad y en parte al crecimiento en el incremento de propiedades en el extranjero (véase Ryan 1989). Es asimismo probable que estos factores obliguen a los operadores turísticos a buscar un mayor control de calidad de todos los aspectos de las vacaciones, algo que hasta ahora no han logrado: vale la pena señalar que apenas el doce por ciento de los visitantes extranjeros a Gran Bretaña estaban en una vacación inclusiva (página web de Key Note). Barrett (1989b) también sugiere que parte del traslado a los viajes independientes "se debe en parte a una reacción a la "lo poco de moda" ["naffness"] de las vacaciones empaquetadas", que ya en la década de 1980 eran vistas como algo pasado de moda o no inteligente.

Hasta el momento he examinado algunas caracteristicas de la industria referidas al transporte de vacacionistas británicos y de Europa septentrional, fundamentalmente a ciertos paises del sur europeo dentro de los cuales hay una gran concentración espacial (véase Goodall 1988: 25-26). En la siguiente sección consideraré la organización de la industria turistica en sociedades que reciben un gran número de estos visitantes. Pero antes de ello examinaré algunos rasgos de esta industria, referidos al suministro de servicios dentro de Gran Bretaña. 
John Urry: La cambiante economía de la Industria del turismo

Hay un inmenso mercado potencial en lo que a visitantes extranjeros respecta. A comienzos de la década de 1980 apenas el siete por ciento de los ciudadanos estadounidenses tenía pasaporte; esta cifra sólo tuvo que llegar al diez por ciento para que hubiese un incremento sumamente grande en el número de visitantes estadounidenses potenciales (véase Cabinet Office 1983). Las preferencias de los visitantes extranjeros son altamente localizadas, siendo la mayoria de ellos atraidos a Londres y a diversos pequeños pueblos y ciudades del interior. De hecho, alrededor del ochenta por ciento iniciaba su visita en la capital y eso se reflejaba en, por ejemplo, que una alta proporción de quienes visitaban los teatros del West End londinense eran extranjeros (SWET 1982). Su incremento en Londres no produjo ningún desvio significativo a otros centros provinciales que no atrajesen ya a un número importante de los visitantes. Es màs, podria muy bien ser que de no darse ningún giro en los patrones de los mismos, y si la oferta de espacio hotelero en Londres permanece baja, entonces los visitantes extranjeros se dirigirán a otros destinos en Europa antes que al resto de Gran Bretaña. En la década de 1980 se calculó que no hubo ningún incremento en el número de visitantes nuevos al Reino Unido, y que todo el incremento se habia dado en visitantes "repitentes". Dichas "repeticiones" son más comunes entre los viajeros de negocios, europeos occidentales, visitantes de más de treinta y cinco años de edad, y personas que viajan afuera de Londres (BTAVETB Research Services 1988).

Las seis actividades de esparcimiento más populares de los visitantes extranjeros son visitar tiendas o mercados (82 por ciento), restaurantes o cafés (77 por ciento), iglesias, catedrales y asi por el estilo (69 por ciento), lugares/edificios históricos (69 por ciento), museos y galerias de arte (64 por ciento), y ciudades 0 pueblos históricos (62 por ciento) (BTAVETB Research Services 1988). En 2000 hubo el asombroso número de 28 millones de visitas del extranjero a Londres, esperándose que las noches de visitante superen los 120 millones (página web de la Global Hotel Network). A continuación consideraré una serie de otras caracteristicas de la organización económica y geográfica del turismo británico.
En 1999 había 288,000 establecimientos en la industria de hospedaje británica, lo que generaba un movimiento anual de $£ 43$ billones (página web del Department of Culture, Media and Sport). Con respecto a los hoteles, la mayor parte del alojamiento se brinda en pequeñas unidades. En la década de 1980 se calculó que del medio millón de habitaciones de hotel, el treinta por ciento era de propiedad corporativa, en tanto que el setenta por ciento era de independientes, la mayoría de ellos con menos de cincuenta habitaciones (Slattery y Roper 1986). El pequeño capital tiene, entonces, un significado duradero en el suministro de alojamiento para los turistas en Gran Bretaña. Resulta útil distinguir entre estos pequeños capitales a cuatro sectores diferentes.

Están los "auto-empleados", sin trabajo de terceros y dependientes del trabajo familiar y las habilidades basadas en el oficio. Son económicamente marginales, las habilidades gerenciales formales son débiles y no hay un divorcio entre la propiedad y el control. Muchas unidades de cama-y-desayuno son de este tipo. En segundo lugar tenemos la categoria de "pequeños empleadores", que se distinguen de los autoempleados por el empleo intermitente de trabajadores externos. Muchas casas de huéspedes y pequeños hoteles son de este tipo. En tercer lugar están los "propietarios controladores" que no utilizan mano de obra familiar sino trabajadores externos, los que pueden recibir un considerable grado de capacitación. Los niveles de inversión de capital son más elevados y hay medios más formales de control administrativo. Son menos marginales económicamente, aunque no hay un divorcio entre la propiedad y el control. Son de este tipo muchos de los llamados hoteles de "casa de campo", donde el propietario está directamente involucrado. Por último están los "dueños directores", donde existe una considerable inversión de capital, capacitación formal y la separación de la propiedad y el control. Este es el tipo más común entre los hoteles en el centro de las ciudades que no forman parte de ninguna cadena.

Todos estos distintos tipos de pequeños capitales en la industria hotelera y de abastecimiento de servicios muestran una enorme vulnerabilidad al mercado. Una cuarta parte de estos establecimientos cierra en dos años, la mitad en 
cuatro. Aproximadamente la cuarta parte si sobrevive, en tanto que los restante tres cuartos se encuentran en un continuo estado de flujo. La tasa de rotación es considerablemente más elevada en esta industria que en la mayoria, lo que significa que hay una gran inseguridad laboral. La categoria de "propietarios controladores" parece ser la que tiene mejores condiciones para sobrevivir. Drew Smith (1988: 22), ex-editor de The Good Food Guide [La guia de la buena comida], sostiene que "el restaurante [y el hotel] administrado personalmente es el que ha resistido la tendencia, a menudo por la sola dedicación del dueño. En el otro extremo del mercado, esto usualmente se debe a que la familia ha conservado el control absoluto de la propiedad". Por lo tanto, esta es una industria de una enorme volatilidad. Y si bien hay, como veremos, unos cuantos operadores sumamente grandes, hay muy pocas restricciones para el ingreso (iy la salida!). Drew Smith estima que cincuenta nuevos restaurantes abren cada semana en Londres. En efecto, ciertamente hay grandes variaciones en la propensión a abrir nuevos restaurantes y hoteles en distintas partes del país. Cornwall, por ejemplo, parece haber tenido tradiciones relativamente débiles del empresariado local en todos los sectores de la economia, el turismo inclusive, aunque la mayoría de las empresas relacionadas con este último son en realidad pequeñas. En un estudio de Looe se halló que había un nivel sumamente elevado de movilidad geográfica entre los empresarios (Hennessy et al. 1986: 16). En general, Cornwall atrae empresarios en lugar de generar los suyos.

Una influencia importante en los tipos de actividades relacionadas con el turismo halladas en distintas zonas (Londres en comparación con Looe), es el crecimiento del turismo de negocios y su impacto diferencial. El turismo empresarial está conformado por una serie de componentes: reuniones efectuadas en otras partes del pais, conferencias y la concesión de viajes como una gollería no afecta a impuestos (véase Williams y Shaw 1988b: 19). Se ha dado, en particular, un gran incremento en el número de conferencias con casi un millón de delegados que anualmente asisten a ellas (British Hospitality Association 2000). El efecto de las conferencias y del turismo empresarial, en particular, ha generado un periódico boom de cons- trucción hotelera, por ejemplo en Londres a finales de la década de 1960 y comienzos de la de 1970 , en la de 1980 y finales de la de 1990. Veintitrés nuevos hoteles abrieron alli en 2000 , estando asombrosamente proyectados unos setenta y ocho (página web de Global Hotel Network; para detalles de los grupos hoteleros véase British Hospitality Association 2000).

Un desarrollo significativo en los últimos diez años aproximadamente fue el desarrollo de los consorcios hoteleros. Éstos surgieron en la década de 1960 para compensar la estructura descentralizada del capital y los problemas que ello creaba para su acumulación y la reducción sistemática de costos. El crecimiento corporativo resulta difícil dados los bajos niveles de concentración, el bajo tamaño por unidad y la combinación variable de comidas, bebidas y alojamiento, cuya demanda es tanto estacional como volátil (véase Litteljohn 1982; Slattery et al. 1985; Bagguley 1987). Los consorcios permiten a los hoteles alcanzar economias de escala y con ello pueden competir con mucha mayor efectividad con las economías al alcance de las grandes compañias antes enumeradas. Se dividen en varios tipos:

- Consorcios de mercado: para brindar acceso a un departamento de mercadeo corporativo, sobre la base ya sea de un agrupamiento regional o de un segmento específico del mercado (como Prestige)

- Consorcios de mercadeo y compras: además de mercadear economias también negocian precios reducidos para pedidos grandes

- Consorcios referenciales: para suministrar un sistema nacional o internacional de referencias, conectadas en particular con las aerolíneas

- Consorcios de personal y capacitación: para proveer capacitación y funciones de personal en común

- Sistemas de reserva: para suministrar un sistema de reservas nacionales o internacionales, vinculadas a menudo con varias otras oficinas de turismo

La internacionalización del suministro del esparcimiento se da en muchas partes de 
los servicios relacionados con el turismo. Esto se puede ver en forma más clara en el caso de McDonalds, con sus 23,300 restaurantes y su reciente ingreso a los hoteles Golden Arches de Suiza). La atracción turistica más importante de Europa es Disneylandia (Paris), que abrió en 1992. Ella atrae a doce millones de visitantes al año con 43 atracciones y 14 horas de espectáculos en vivo. Acaba de abrir un inmenso centro comercial y un segundo parque -Disney Studios- está a punto de levantarse en el mismo lugar (página web de Disneylandia Paris).

Entonces, las industrias relacionadas con el turismo aqui analizadas son intensamente competitivas. Es más, aunque los operadores muy grandes pueden desplazar su capital de un lugar a otro en respuesta a las cambiantes presiones del mercado, la mayor parte de la industria no puede moverse, y ciertamente no en el corto plazo. Ella tiene una gran fijeza espacial, un grado excepcional de descentralización y una inmensa volatilidad en el gusto. Dado que muchos visitantes de hoteles y restaurantes sólo están alli por las atracciones disponibles en la zona, puede muy bien haber un gran respaldo a la mejora de las instalaciones y servicios locales, y a incrementar el número o atractivo de los posibles objetos de la mirada del turista. De ahí que el capital relacionado con el turismo puede muy bien estar a favor de las grandes inversiones públicas de parte de las autoridades locales, para que suministren objetos nuevos 0 mejorados que los visitantes puedan ver.

En décadas anteriores, las compañias involucradas en la industria de la hospitalidad siguieron diversas estrategias de reducción de costos:

1)La expansión del alojamiento autoservido.

2 )El extenso uso de la tecnología de la información. Ello se debe a que los recursos no utilizados de la industria, como una cama de hotel, no pueden almacenarse; a la inmensa volatilidad de la demanda; $y$ al hecho de que las unidades de turistas están necesariamente dispersas geográficamente. La Internet, en particular, permite que se establezcan diversos tipos de red entre los consumidores potenciales y las mu- chas unidades de abastecimiento locales, específicas y descentralizadas.

3)Cambiando el insumo trabajo, en particular mediante el crecimiento del empleo femenino a tiempo parcial.

4)Economizando los costos uniéndose a un grupo o consorcio.

5)Cerrando en los periodos de poca actividad o alternativamente, intentando generar negocios extras durante la temporada baja; desarrollando "temporadas de apoyo" "shoulder seasons".

6) Generando un ingreso extra mejorando la calidad del producto suministrado, como mejores comidas, trabajadores mejor preparados, o servicios en la habitación.

\section{El impacto del turismo en el extranjero}

El desarrollo del turismo fuera del Reino Unido ha tenido un amplio impacto económico, social y cultural. Basándome en parte en el concepto del bien posicional antes discutido, consideraré algunos países mediterráneos, a lo que seguiré con algunos comentarios sobre Norteamérica y el sudeste asiático.

Existen unas relaciones complejas entre los turistas y las poblaciones nativas de los lugares a los que aquellos miran; la artificialidad resultante de muchas atracciones turísticas se debe a la naturaleza particular de las relaciones sociales que se establecen en dichos lugares entre "anfitriones" y "huéspedes" (con respecto a lo que sigue véase Smith 1989). Hay una serie de determinantes de las relaciones sociales particulares establecidas entre ambos.

1)El número de turistas que visitan un lugar en relación a las dimensiones de la población anfitriona y a la escala de los objetos que se está mirando. Por ejemplo, el tamaño geográfico de Nueva Zelanda permitiria que más turistas la visiten sin un daño medioambiental o un efecto social indeseable. En cambio, la pequeñez física de Singapur significa que los turistas adicionales no pueden ser alojados con facilidad, salvo con la construcción de 
más hoteles, lo que sólo sería posible demoliendo las pocas tiendas chinas que aún quedan y que fueron uno de los principales objetos de la mirada turística. Del mismo modo, la ciudad medieval de Dubrovnik tiene un límite fisico absoluto, determinado tanto por las murallas de la ciudad como por la población de más de cuatro mil personas que actualmente vive allí.

2)El objeto predominante de la mirada turística, ya sea un paisaje rural (el Lake District), uno urbano (Chester), un grupo étnico (maories en Rotorua, Nueva Zelanda), un estilo de vida (el "lejano oeste"), artefactos históricos (la catedral de Canterbury o Wigan Pier). las bases de la recreación (los campos de golf en St. Andrews), o simplemente la arena, el sol y el mar (Mallorca). Aquellas actividades turisticas que involucran la observación de objetos físicos son menos intrusas que las que comprenden la observación de personas y grupos. Es más, en esta última categoria la observación de la vida privada de los grupos anfitriones producirá el estrés social mayor. Entre los ejemplos de este tipo tenemos a los esquimales o a los masai, que respondieron a la mirada cobrando una " $E$ por carro" en cada visita a sus chozas de barro. En cambio, alli donde lo que se observa es más un ritual público, el estrés social será menos pronunciado y de hecho se favorecerá positivamente una participación más amplia, como en varios de los rituales balineses (véase Smith 1989: 7).

3 ) La naturaleza de la mirada involucrada y el "empaque" espacial y temporal resultante de los visitantes. Por ejemplo, la mirada podría ser algo que puede darse en forma más o menos instantánea (ver/fotografiar Mount Cook, la montaña más alta de Nueva Zelanda), o tal vez necesite una exposición prolongada (ver/experimentar el "romance" de París). En el primer caso, los turistas japoneses pueden volar para una visita que dura apenas unas cuantas horas, en tanto que la experiencia del romance parisino requerirá una inmersión más prolongada y "profunda".

4 )La organización de la industria que se desarrolló para servir a la mirada masiva: si es de propiedad y financiamiento privado o público; si es de propiedad local o si involucra intereses extranjeros; si el capital involucrado es predominantemente pequeño o grande; y si hay conflictos entre la población local y la emergente industria turística. Estos conflictos pueden darse en torno a muchos temas: la conservación en oposición al desarrollo comercial, los salarios a pagarse a los empleados reclutados localmente, los efectos que el desarrollo tiene sobre las costumbres y la vida familiar locales, lo que podriamos llamar el "abaratijamiento" de las artesanías locales y cómo compensar la estacionalidad esencial del trabajo (véase Smith 1989).

5 )Los efectos del turismo sobre las actividades agrícolas e industriales preexistentes. Ellos pueden abarcar desde la destrucción de dichas actividades (buena parte de la agricultura en Corfú) a su erosión gradual a medida que la mano de obra y el capital son llevados al turismo (partes de España), o su preservación al intentarse salvar las actividades preexistentes como otros objetos más que mirar (la ganaderia, y por lo tanto el pastoreo, en Norfolk Broads).

6 )Las diferencias económicas y sociales entre los visitantes y la mayoria de los anfitriones. En Europa septentrional y Norteamérica, el turismo crea menos tensiones dado que la masa de "anfitriones" serán ellos mismos "invitados" en otras ocasiones. Podría suceder que el turismo desarrolle en forma desordenada el "entendimiento internacional". Es dificil explicar el giro en la opinión pública de Gran Bretaña hacia un pro-europeismo en la década de 1980 , sin reconocer que algún papel le cupo a la industria europea del turismo y la forma en que los grandes 
flujos de visitantes hicieron que el continente fuera familiar y no amenazante. Sin embargo, en otros lugares usualmente hay enormes desigualdades entre los visitantes y la población indigena, la vasta mayoria de la cual jamás podría imaginarse a sí misma contando ya sea con el ingreso o el tiempo libre como para a su vez ser turistas. Estas diferencias se refuerzan en muchos paises en vias de desarrollo con la naturaleza del avance turisti$\mathrm{co}$, que parece ser excepcionalmente opulento y altamente capitalizado, como por ejemplo en muchos hoteles de India, China, Singapur, Hong Kong y el norte de África, en parte porque hay tan pocos servicios disponibles, tanto para los visitantes como para la población anfitriona.

7 JEl grado en que la masa de visitantes exige estandares particulares de alojamiento y servicio, que deban ser encerrados en una burbuja ambiental para protegerles de muchas caracteristicas de la sociedad anfitriona. Esta demanda es más marcada entre los visitantes de los tours inclusivos, quienes no solamente esperan niveles occidentales de alojamiento y alimentación. sino también un personal bilingüe y arreglos bien orquestados. Estos turistas rara vez dejan la seguridad de la burbuja turistica occidental y en cierta medida son tratados como "niños" dependientes por los turistas profesionales (vèase Smith 1989: 1011). En algunos casos la cultura indigena es realmente peligrosa, como en Sicilia, algunas partes de Nueva York y recientemente en Florencia. Esta demanda es menos pronunciada entre los "viajeros" exploradores individuales, los turistas más pobres como los estudiantes, y aquellos visitantes para los cuales "jugársela" forma parte de la experiencia que esperan como turistas (para el turismo mochilero véase Edensor 1998).

8 )El grado en que el estado de un pais dado busque promover activamente desarrollos turísticos, o que alternati- vamente se esfuerce por prevenirlos. Buenos ejemplos del primer caso son España, Túnez y Hawai, todos los cuales están desarrollando activamente una cultura turistica plena, en la cual el gran número de turistas ha pasado a formar parte del "escenario regional" (Smith 1989). En cambio, muchos de los estados petroleros tienen razones morales/sociales decididas explicitamente para restringir el turismo negando visas (Arabia Saudita es un buen ejemplo de ello). Del mismo modo, durante la Revolución Cultural en China el estado buscó activamente prevenir su crecimiento. Cuando esto cambió entre comienzos y mediados de la década de 1970, los visitantes occidentales eran tan raros que a menudo se les aplaudía como si fueran miembros de la realeza.

9 )El grado en que los turistas puedan ser identificados y culpados por efectos económicos y sociales supuestamente indeseables. Esto es obviamente más común cuando dichos visitantes son económica y/o cultural y/o étnicamente diferentes de la población anfitriona. Es asimismo más común cuando esta última viene experimentando rápidas transformaciones económicas y sociales. Sin embargo, dichos cambios no necesariamente son resultado del "turismo". Por ejemplo, en el caso de Tonga, lo que explica la alta tasa de inflación no es el flujo anual de visitantes sino más bien la gran sobrepoblación. Y sin embargo, es por supuesto mucho más fácil atribuir los problemas indígenas de desigualdad socioeconómica al "extranjero sin nombre y sin rostro" (véase Smith 1989). Es más, algunas objeciones locales al turismo son, en realidad, objeciones a la "modernidad" misma: a la movilidad y al cambio, a nuevas formas de interrelación personal, a un papel reducido de la familia y la tradición, y a diferentes configuraciones culturales (véase el "Código global de ética del turismo" en la página web de la Organización Mundial de Turismo). 
De este modo, el impacto social del turismo dependerá de la intersección de una amplia gama de factores. Por ejemplo, se ha expresado gran preocupación con respecto a sus probables consecuencias en diversos paises mediterráneos. Su crecimiento en esta zona es uno de los desarrollos económicos y sociales más significativos de la postguerra. Es un simbolo particularmente llamativo de la reconstrucción de Europa occidental en este periodo.

Hay una gran elasticidad de ingreso en la demanda de los servicios turísticos, y como el ingreso ha crecido en Alemania Occidental, Francia, Escandinavia, los Paises Bajos y las Islas Británicas, se ha dado un incremento más que correspondiente en la demanda de los viajes al extranjero. De hecho, Europa occidental daba cuenta del 68 por ciento de todos los turistas internacionales (Williams y Shaw 1988a: 1). En respuesta a esta demanda, los paises de Europa meridional desarrollaron enormes industrias turisticas. $Y$ ellas han sido especialmente costo-efectivas, lo que a su vez ha bajado los costos reales del viaje al extranjero y llevado con ello a una ampliación aun mayor de la demanda. España fue el primero y ha seguido siendo el más grande de los destinos en el Mediterráneo. Otros destinos importantes son Francia, Italia, Grecia, Portugal y Turquia. En 1984, los ingresos procedentes del turismo daban cuenta del cuatro por ciento del ingreso nacional de España y Portugal, más del tres por ciento del de Grecia, y del 2.6 por ciento del de Italia (Williams et al. 1986: 13). En términos globales, el turismo genera una redistribución neta de la riqueza del norte al sur de Europa, en especial a España, Portugal e Italia.

Son bien conocidos los efectos problemáticos que estos desarrollos turísticos han tenido en por lo menos algunos de los países. Ellos se deben al gran número de turistas y su demanda estacional de servicios; los nocivos efectos sociales debidos, sobre todo, a la disponibilidad de trabajo por género, la concentración geográfica de los visitantes, la ausencia de una politica de respuesta concertada, las diferencias culturales entre anfitriones y huéspedes, y a la exigencia hecha por muchos visitantes de ser encerrados en "burbujas ambientales". Un lugar "inundado" de turistas es Florencia, donde la población residente de 500,000 personas dio cabi- da a 1.7 millones de visitantes anuales en la década de 1980. Esto llevó a la propuesta, presentada en esa década, de retirar del centro de la ciudad sus funciones académicas, comerciales e industriales y dedicar Florencia integramente al turismo. Según los críticos, ello habría significado su "disneyficación" (Vulliamy 1988: 25).

Robert Graves (1965: 51) rechazó la transformación turística similar de Mallorca:

La vieja Palma hace tiempo dejó de existir, engullido su centro por los restaurantes, bares, tiendas de recuerdos, agencias de viaje y cosas parecidas ... Inmensas y nuevas conurbaciones han surgido a lo largo de la costa vecina ... El principal uso dado a los árboles de oliva parece ser su conversión en ... ensaladeras y cajas que vender a los turistas. Pero como señalase un bromista de Mallorca, una vez que todos hayan sido cortados tendremos que levantar otros de plástico para que los turistas los admiren desde las ventanas de sus ómnibus.

Se ha sugerido que existe una amenaza sumamente seria a toda la costa mediterránea. Éste es el destino turístico más popular del mundo. Un informe de la ONU sugiere que el número de visitantes podría incrementarse de cien millones en la década de 1980 a 760 millones en 2025, generando así una presión enorme sobre los recursos alimenticios, hídricos y humanos. El crecimiento de las ciudades costeras necesita desacelerar su ritmo dramáticamente (Guardian, 2 de noviembre de 1988). Pero lo opuesto viene sucediendo con Turquía, un país que recientemente se desarrolló como destino turístico importante. La atracción inmediata para los inversionistas locales alli es que la mayor parte de los ingresos provienen en forma de divisas extranjeras. Hasta ahora, el turismo turco ha involucrado la proliferación de algunos horribles proyectos no planificados, como aquellos en Bodrum y Mármara, que tal vez tengan que ser demolidos bastante pronto. Simply Turkey [Simplemente Turquia], un operador especializado, dejó de vender vacaciones en Gumbet porque "ya no era pequeño y hermoso; es un lugar de edificios desparramados, ruidoso y pol- 
voriento, con una playa que no es lo bastante grande como para atender su rápido desarrollo" (citado en Whitaker 1988: 15). El impacto de un crecimiento turístico tan rápido se siente en forma particularmente aguda porque el sudoeste turco siempre ha atraido un número considerable de "viajeros" individuales gracias a la cualidad excepcional de sus antigüedades. Por lo tanto, Turquia se encuentra en medio de los intereses conflictivos del turismo de masas y otro más socialmente selectivo, y entre las miradas turisticas colectiva y romántica.

La segunda área más importante de actividad turistica a nivel mundial es América del Norte. Resulta interesante que los desarrollos acá sean diferentes de los de Europa. Algo central para el turismo norteamericano fueron el automóvil, la autopista, la vista a través del parabrisas y la avenida comercial. Jackle (1985: cap. 9 para lo que sigue) señala como, en la postguerra, las ciudades, pueblos y áreas rurales fueron todas rehechas en lo que él llama el "orden universal de la autopista". En 1950, el 80 por ciento de todos los viajes de larga distancia fueron hechos en automóvil, y para 1963 el 43 por ciento de todas las familias estadounidenses tomaron largos viajes de vacaciones cada año, promediando 600 millas.

Hubo una rápida mejora en la calidad del sistema vial para que pudiera atender a los viajes más veloces y a un volumen más grande de tráfico. Infortunadamente había poco que ver en las nuevas carreteras, salvo por la monotonía de la misma autopista. John Steinbeck dijo que "será posible viajar de Nueva York a California sin ver una sola cosa" (citado en Jakle 1985: 190). Las carreteras fueron construidas por la conveniencia del manejo, no por los patrones de la vida humana que se pudieran generar. La ubicuidad de la radio en los automóviles estadounidenses, y en cierta medida del aire acondicionado, aisla a los pasajeros de casi todos los aspectos del medio ambiente, salvo de la vista que se tiene a través del parabrisas (Sheller y Urry 2000).

$Y$ esta vista no revela casi nada porque hasta los paisajes urbanos constan de calles comerciales, la erradicación aleatoria de lugares distintivos y la generación de un paisaje estan- darizado. Jackle llama a esto la producción de la "lugarcomunidad". Las calles comerciales son lugares comunes que carecen de las ambigüedades y complejidades que usualmente hacen que un sitio sea interesante. Son "paisajes unifuncionales" que toman un aspecto aún más uniforme a medida que las grandes corporaciones administran cadenas de establecimientos parecidos y estandarizados ( $M c D$ onalds, Howard Johnson, Col. Saunders, Holiday Inn y asi sucesivamente). El viaje en automóvil ha pasado a ser uno de los íconos de la América de la postguerra, como se refleja en On the Road, de Kerouac, o en la película Easy Rider. En Lolita, Humbert Humbert concluye que "hemos estado en todos lados. No vimos nada" (citado en Jackle 1985: 198).

Uno de los lugares turísticos más famosos de Norteamérica es las Cataratas del Niágara. Las reacciones que provoca siempre comprende superlativos (véase Shields 1990). Los observadores decian no encontrar las palabras adecuadas. Era una maravilla exótica y tenia una inmensa aura natural. Sin embargo, una serie de transformaciones que han tenido lugar han convertido al Niágara en una serie de distintos objetos de la mirada turística. En primer lugar, en el tardío siglo XIX las cataratas pasaron a ser el lugar preferido para las lunas de miel y para el cortejo en general. Shields vincula esto con la forma en que ellas conformaban una admirable zona liminal, en la cual las estrictas convenciones sociales de la burguesía se relajaban con las exigencias de los viajes y el anonimato relativo. La asociación histórica de las cataratas con la pasión, ya fuera el amor o la muerte, incrementó aún más la prominencia de dicha zona. Los viajeros esperaban que ellas fueran excepcionales, un lugar donde se trascendiesen los límites de una experiencia ordinaria. El viaje era análogo a un peregrinaje. Nathaniel Hawthorne señaló haber quedado "encantado con una visión de espuma y furia, y acantilados vertiginosos, y un océano que se precipita desde el cielo" (citado en Shields 1990). Sin embargo, más recientemente la luna de miel quedó vaciada de su simbólico status liminal. Ella ha pasado a ser un cliché nupcial sin sentido, referido a nada sino a si mismo. Todo el énfasis en las cataratas cae en los tinglados, en las suites de luna de miel y en las "tinas del amor" en for- 
ma de corazón. Las cataratas ahora representan lo kitsch, el sexo y el espectáculo comercial. Es como si ya no se encontraran alli y solamente pudiera vérselas a través de sus imágenes.

De este modo, el mismo objeto en un sentido fisico ha sido transformado por diversos intereses comerciales y públicos. La naturaleza de la mirada ha experimentado enormes cambios. En el siglo XVIII las cataratas eran un objeto con una intensa aura natural; en el siglo XIX funcionaban como una zona liminal a ser mirada y experimentada profundamente por las parejas que se cortejaban; $y$ en el tardío siglo $X X$ han pasado a ser otro "lugar" a coleccionar por el visitante inmensamente móvil, para quien su contemplación representa un espectáculo, el sexo y un desarrollo comercial.

Un tipo de desarrollo afín fue el crecimiento del llamado "turismo sexual" en el sudeste asiático. En Corea del Sur esto fue fomentado especificamente por el estado. Su forma principal consiste en el tour kisaeng,(3) diseñado concretamente para los empresarios japoneses (véase Mitter 1986: 64-67). Muchas compañias japonesas recompensan a su personal masculino sobresaliente con giras a burdeles y fiestas de kisaeng con todos los gastos pagados. Los ministros surcoreanos han felicitado a las "chicas" por su contribución al desarrollo económico de su pais. Las Filipinas y Tailandia son otros paises con una industria sexual igualmente floreciente. En el primer caso el estado promueve el uso de "muchachas de hospitalidad" en el turismo, y el ministerio del ramo recomienda diversos burdeles (Mitter 1986: 65). Los paquetes de tours organizados conjuntamente con un agente en Manila incluyen "muchachas de hospitalidad" preseleccionadas. Alrededor del siete-ocho por ciento del dinero ganado será retenido por las mujeres mismas. Se calcula que en Tailandia hay unas 500,000 de ellas que trabajan en la industria sexual, con tal vez unas 200,000 nada más en Bangkok (véase Lea 1988: 66-69). Los procesos específicos que ayudaron a generar dicho patrón son: el conjunto excepcionalmente fuerte de prácticas patriarcales que pintan a las mujeres ya sea como "madonnas/virgenes" o

13) Originaimente la contraparle coreana de las geishas japonesas, las kisaeng desaparecieron a mediados del siglo XX. pero actualmente las prostitutas de lujo han optado por llamarse asi "putas"; la creencia en las personas de los países afluentes de que las mujeres de color son más asequibles y sumisas; la elevada tasa de incesto y violencia doméstica de parte de padres/esposos en algunas de estas sociedades; la despobla ción rural que atrae personas a las ciudades en busca de cualquier trabajo posible; y el crecimiento de compañias de viaje y páginas web "especializadas", dedicadas a facilitar el viaje de grupos de "turistas sexuales" varones (véase Enloe 1989, sobre todo en torno a los intentos efectuados por las mujeres para organizar grupos que protejan a las prostitutas; Clift y Carter 1999).

Unos factores similares se aplican en otros lugares, pero tales patrones son menos evidentes. Singapur presenta un contraste interesante. En los materiales publicitarios para los turistas no hay referencias al turismo sexual. Los únicos clubes listados son diversas discotecas y espectáculos de estilo asiático. Sin embargo, Singapur es un objeto extremadamente exitoso de la mirada turística, pero esto se ha logrado des-enfatizando su naturaleza exótica. Buena parte del énfasis en los materiales publicitarios se refiere a sus atractivos como un moderno centro comercial, y efectivamente hay un extraordinario complejo de centros comerciales a lo largo del ahora mal llamado Orchard Road [Camino del Vergel]. Singapur también ha transformado muchas de las viejas zonas de tiendas chinas en modernos complejos hoteleros, entre ellos el que se dice es el hotel más alto del mundo, asi como el modernizado Hotel Raffles. Singapur está "en el este" pero en realidad ya no es parte "del este". Es casi la ciudad moderna máxima y no se presenta a si misma a los visitantes como "exótica/erótica".

\section{Conclusión}

Es claro que los efectos del turismo son sumamente complejos y contradictorios, dependiendo de la gama de consideraciones amiba esbozadas. Nada sorprendentemente, han habido muchas discusiones sobre cuán deseable es el turismo como una estrategia de crecimiento económico en las llamadas sociedades en vías de desarrollo. Esto plantea muchas cuestiones dificiles. 
El crecimiento del turismo en los países en vías de desarrollo, como el "turismo de cacería" en Kenya, el "turismo étnico" en México, el "deportivo" en Gambia y asi sucesivamente, no se deriva simplemente de los procesos internos de dichas sociedades. Semejante posibilidad de desarrollo se debe a una serie de condiciones externas: cambios tecnológicos tales como los viajes aéreos baratos y los sistemas de reserva de pasajes computarizados; los desarrollos dados en el capital, entre ellos el crecimiento a nivel mundial de grupos hoteleros (Ramada), agencias de viaje (Thomas Cook) y organizaciones de finanzas personales (American Express); la difundida penetración de la mirada "romántica", de modo tal que más y más personas desean aislarse del patrón existente del turismo de masas; la creciente fascinación del primer mundo con las prácticas culturales de las sociedades menos desarrolladas; la aparición del turista esencialmente como un "coleccionista" de lugares a menudo contemplados y experimentados superficialmente; y el surgimiento de un poderoso lobby metropolitano dedicado a promover la posición de que el turismo tiene un gran potencial de desarrollo (véase Crick 1988: 47-48).

Los beneficios económicos del turismo a menudo son menores de lo esperado. Buena parte de las inversiones hechas en él en el mundo en desarrollo fueron en realidad efectuadas por compañias de gran escala con sede en Norteamérica o Europa occidental, y el grueso de los gastos en turismo son retenidos por las compañias transnacionales involucradas; a menudo apenas el veinte-sesenta por ciento del precio queda en el pais anfitrión (página web de Tourism Concern). Por ejemplo, en Mauricio el noventa por ciento de las divisas extranjeras ganadas con el turismo son repatriadas a compañías con sede en otro lugar. Con el alto nivel actual de integración vertical de la industria, resulta sumamente probable que esta repatriación se dé (véase Crick 1988: 45).

Otro problema más, también evitado en Singapur, aparece cuando el turismo da cuenta de una proporción excepcionalmente alta del ingreso nacional del pais. Algunas islas caribeñas experimentan esta dificultad. Eso quiere decir que se perderá una parte enorme del ingreso nacional si algo ayuda a minar la demanda turística. Esto es lo que sucedió en 1987 en Fiji, por ejemplo, luego de los golpes militares (véase Lea 1988: $32-36$, en especial sobre la escala de la publicidad necesaria para restaurar la confianza de los consumidores, fundamentalmente en Australia).

También debe preguntarse: ¿desarrollo para quién? Muchas de las instalaciones y servicios debidas al turismo (aeropuertos, campos de golf, hoteles de lujo y así sucesivamente) serán poco beneficiosas para la masa de la población indígena. De igual modo, buena parte de la riqueza indígena local que se genere se distribuirá en forma sumamente desigual, de modo que la mayoría de la población de los países en desarrollo obtendrá pocos beneficios. Esto depende, claro está, de los patrones de propiedad local. Por último, bastantes de los empleos generados por los servicios relacionados con el turismo son relativamente poco calificados y pueden muy bien reproducir la naturaleza servil del régimen colonial anterior, lo que un crítico denominó la "preparación de lacayos" (citado en Crick 1988: 46).

Sin embargo, debemos preguntarnos si muchos países del Tercer Mundo tienen muchas alternativas al turismo como estrategia de desarrollo. Aunque existen serios costos económicos, asi como sociales que aqui no he considerado, ante la ausencia de alternativas resulta sumamente difícil ver que estas sociedades tengan muchas opciones fuera de desarrollar su atractivo como objetos de la mirada turística, en particular para visitantes de Norteamérica, Europa occidental y cada vez más del Japón.

En el siguiente capítulo consideraré un punto que acabo de mencionar: la naturaleza de los empleos a encontrar en servicios relacionados con el turismo, una cuestión importante tanto en los países en vías de desarrollo como en los ya desarrollados, ya que éste da cuenta de una proporción cada vez mayor del empleo asalariado en todo el mundo. 


\section{Bibliografía}

BAGGULEY, P.

1987 Flexibility, Restructuring and Gender. Changing Employment in Britain's Hotels. Lancaster Regionalism Group Working Paper no. 24.

\section{BARRETT, $F$}

1989 'Why the tour operators may face their last supper', The Independent, 7 Noviembre

BARTHES, R.

1972 Mythologies. Londres: Jonathan Cape

BECKERMAN, $W$.

1974 In Defence of Economic Growth. Londres: Jonathan Cape

Bourdieu, $P$.

1984 Distinction. Londres: Routledge \& Kegan Paul.

\section{BRITISH HOSPITALITY ASSOCIATION}

2000 British Hospitality: Trends and Statistics. Londres: British Hospitality Association.

BTA/ETB Research Services

1988 Overseas Visitor Survey. Londres: BTAETB Research Services

BTA

2000 Digest of Tourism Statistics No 23 . Londres: British Tourism Authority

CABINET OFFICE (Enterprise Unit)

1983 Pleasure, Leisure and Jobs. The Business of Tourism. Londres: HMSO.

CHANDLER, $P$.

2000 'The UK Outbound Tour Operating Market - Changing Patterns of Distribution', ETC Insights, Londres: English Tourism Council.

CLIFT, S. y CARTER, S. (eds)

1989 Tourism, Travel and Sex. Londres: Cassell.

CRICK, M.

1988 'Sun, sex, sights, savings and servility', Criticism, Heresy and Interpretation. 1: 37-76.

DESFORGES, L.

1998 "'Checking out the planet ". Global representantions/local identities and youth tra- vel' en T. Skelton y G. Valentine (eds) Cool places. Londres: Routledge. pp. 175-92.

\section{EDENSOR, T.}

1998 Tourists at the Taj. Londres: Routledge

\section{ELLIS, A. y HEATH A.}

1983 'Positional competition, or an offer you can't refuse', en Ellis, A. y Kumar, K. (eds) Dilemmas of Liberal Democracies. Londres: Tavistock. pp. 1-22.

ENGLISH TOURISM COUNCIL 2000/2001 ETC Insights. Londres: ETC.

ENLOE, C.

1989 Bananas, Beaches and Bases. Londres: Pandora

GOODALL, B.

1988 'Changing patterns and structures of European tourism', in B. Goodall y G. Ashworth (eds) marketing in Tourism Industry, Londres: Croom Helm. pp. 18-38.

GRAVES, R.

1965 Majorca Observed. Londres: Cassell.

HENNESSY, S., GREENWOOD, J., SHAW, G. y Williams, $A$.

1986 The Role of Tourism in Local Economies: a Pilot Study of Looe, Cornwall. Tourism in Cornwall Project, Dept of Geography, University of Exeter.

HIRSCH, F.

1978 Social Limits to Growth. Londres: Routledge \& Kegan Paul..

JAKLE, J.

1985 The Tourist. Lincoln: University of Nebraska Press

LEA, J.

1988 Tourism and Development in the Third World. Londres: Routledge.

MAWBY, R., BRUNT, P. y HAMBLY, Z.

2000 'Fear of crime among British holiday makers', British Journal of Criminology, Delinquency and Deviant Social Behaviour, 40: 468-79 en Crimen y Turismo.

MISHAN, E.

1969 The Costs of Economic Growth. Harmondsworth: Penguin 
MITTER, S.

1986 Common Fate, Common Road. Londres: Pluto.

REYNOLDS, $H$.

1988 'Leisure Revolution" prime engines of regional recovery'. The Daily Telegraph 2 Diciembre.

\section{ROCCA, T.}

1989 "Bardot scorns "Black tide of filth" in St. Tropez', the Guardian, 10 de Agosto.

SHELLER, M. y URRY, J.

2000 'The city and the car', International Journal of Urban and Regional Research, 24: 737-57.

\section{SHIELDS. R.}

1990 Places on the Margin. Londres: Routledge SLATTERY, P. y ROPER, A.

1986 The UK Hotel Groups Directory: 1986-7. Londres: Cassell.

SMITH, D.

1988 'A fine old stew in the kitchens', the Guardian. Noviembre 12: 22.

SMITH, V.

1989 Hosts and Guest. The Anthropology of Tourism. Philadelphia: University Of Pennsylvania Press, (primera edición 1978).

SWET (Society of West End Theatres)

1982 Britain at its Best: Overseas Tourism and the West End Theater. Londre: SWET.

TURNER, L. y Ash, J.

1975 The Golden Hordes. Londres: Constable.

URRY, J.

1987 'Some social and spatial aspects of services', Environment and Planning D: Society and Space, 5: 5-26.

URRY, J.

1990 'The consumption of "tourism", Sociology, 24: $23-35$
VULLIAMY, E.

1988 'Squalid renaissance', the Guardian, 16 Abril.

WALTER, J.

1982 'Social limits to tourism', Leisure Studies, 1: $295-304$

WHITAKER, R.

1988 'Welcome to the Costa del Kebab', The Independent, 27 febrero.

WILLIAMS

1988, The Economist, 27 Agosto

WILLIAMS, A. y Shaw, G.

1988a 'Tourism and development: introduction', en A. Williams y G. Shaw (eds) Tourism and Economic Development. Londres: Belhaven Press. pp. 1-11.

WILLIAMS, A. y SHAW, G.

1988b 'Western European tourism in perspective' en A. Williams y G. Shaw (eds) Tourism and Economic Development. Londres: Belhaven Press. pp. 12-38.

WILLIAMS, A., SHAW, G., GREENWOOD, J. y HENNESSY, S.

1986 Tourism and Economic Development: a Review of Experiences in Western Europe, Tourism in Cornwall Project, Dept WTO of Geography, University of Exeter.

2000a Tourism Highlights 2000. Madrid: World Tourism Organisation.

WTO

2000b Yearbook of Tourism Statistics 2000. Madrid: World Tourism Organisation.
John Urry

Universidad de Lancaster,

Reino Unido 